\title{
Methodology for quantum logic gate construction
}

\author{
Xinlan Zhou ${ }^{1,2 *}$, Debbie W. Leung ${ }^{3,2 \dagger}$, and Isaac L. Chuang ${ }^{2 \ddagger}$ \\ ${ }^{1}$ Department of Applied Physics, Stanford University, Stanford, California 94305-4090 \\ ${ }^{2}$ IBM Almaden Research Center, 650 Harry Road, San Jose, California 95120 \\ 3 Quantum Entanglement Project, ICORP, JST \\ Edward Ginzton Laboratory, Stanford University, Stanford, California 94305-4085
}

(October 29, 2018)

\begin{abstract}
We present a general method to construct fault-tolerant quantum logic gates with a simple primitive, which is an analog of quantum teleportation. The technique extends previous results based on traditional quantum teleportation (Gottesman and Chuang, Nature 402, 390, 1999) and leads to straightforward and systematic construction of many fault-tolerant encoded operations, including the $\pi / 8$ and Toffoli gates. The technique can also be applied to the construction of remote quantum operations that cannot be directly performed.
\end{abstract}

\section{INTRODUCTION}

Practical realization of quantum information processing requires specific types of quantum operations that may be difficult to construct. In particular, to perform quantum computation robustly in the presence of noise, one needs fault-tolerant implementation of quantum gates acting on states that are block-encoded using quantum error correcting codes [1, 4 . Fault-tolerant quantum gates must prevent propagation of single qubit errors to multiple qubits within any code block so that small correctable errors will not grow to exceed the correction capability of the code. This requirement greatly restricts the types of unitary operations that can be performed on the encoded qubits. Certain fault-tolerant operations can be implemented easily by performing direct transversal operations on the encoded qubits, in which each qubit in a block interacts only with one corresponding qubit, either in another block or in a specialized ancilla. Unfortunately, for a given code, only a few useful operations can be done transversally, and these are not universal in that they cannot be composed to approximate an arbitrary quantum circuit. To obtain a universal set of gates, additional gates have to be constructed using ancilla states and fault-tolerant measurement. Although these additional gates have been constructed successfully [1, 3, 5, 56, their ad-hoc construction is complicated and is not easily generalized.

Another kind of application in which we are challenged to construct useful quantum operations from a limited set of primitives is in distributed quantum information processing. In this problem, certain kinds of communication between different parties are constrained or prohibited, but prior distribution of standard states may be allowed. For example, quantum teleportation [7] demonstrates how an unknown quantum state can be sent between two parties without sending any quantum information, using only classical communication and prior entanglement. Protocols for distributed state preparation and computation are also known [8], but again, they have been largely constructed by hand and offer neither an explanation of why a particular ancilla state is required nor a systematic path for generalization.

A general framework for addressing such problems has been presented in [9]; it uses quantum teleportation as a basic primitive to enable construction of quantum operations that cannot be directly performed through unitary operations. This framework provides systematic and generalizable construction for an infinite family of fault-tolerant gates, including the $\pi / 8$ and Toffoli gates. It does not, however, lead to circuits equivalent to (or as simple as) prior ad-hoc construction for the same gates.

In this paper, we provide an extension to the teleportation method of gate construction with a similar but simpler primitive, which we call "one-bit teleportation" because it uses one qubit instead of two as ancilla. This method simplifies the construction of [9] and, furthermore, provides strikingly unified construction of the $\pi / 8$, controlledphase, and Toffoli gates. An infinite hierarchy of gates, including the controlled rotations $\operatorname{diag}\left(1,1,1, e^{i 2 \pi / 2^{k}}\right)$ used in the quantum factoring algorithm [11], can be constructed with the present scheme.

*Electronic address: xlz@snow.stanford.edu

${ }^{\dagger}$ Electronic address: wcleung@leland.stanford.edu

‡Electronic address: ichuang@almaden.ibm.com 
The structure of the paper is as follows. First, in Section [II, we define one-bit teleportation, and describe its properties and various guises. Its application to fault-tolerant gate construction is presented in Section III, which is followed in Section IV with specific circuits for the $\pi / 8$, controlled-phase, and Toffoli gates. In Section $\mathrm{V}$, we describe the use of one-bit teleportation to derive the two-bit quantum teleportation protocol and to construct a remote quantum gate. We summarize our results in Section VI.

\section{ONE-BIT TELEPORTATION}

In standard quantum teleportation, Alice performs a joint measurement of the unknown qubit and some ancilla, and sends the classical measurement outcome to Bob, who subsequently reconstructs the unknown state. No quantum operation is performed jointly by Alice and Bob, but they need a certain two-qubit entangled ancilla state. (We refer to any state other than the original unknown qubit state as ancilla state.) The same objective, communicating a qubit, can be accomplished in a simpler manner if Alice and Bob are allowed to perform a quantum gate (such as a controlled-NOT gate, a CNOT) between their respective qubits. In this case, only a single qubit ancilla in Bob's possession is required. We call such a quantum circuit one-bit teleportation, which can be derived using the following facts:

- Fact 1: An unknown qubit state $|\psi\rangle$ can be swapped with the state $|0\rangle$ using only two CNOT gates, as shown in the following circuit:

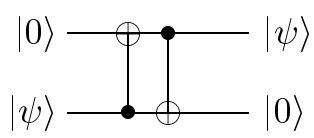

Note that in all circuits we show, time proceeds from left to right as is usual, and conventions are as in [12]. Throughout this section, the first and second qubits refer to the registers with respective initial states $|0\rangle$ and $|\psi\rangle$.

- Fact 2: $X=H Z H$, where $X$ and $Z$ are Pauli operators, and $H$ is the Hadamard gate defined as

$$
H=\frac{1}{\sqrt{2}}\left[\begin{array}{cc}
1 & 1 \\
1 & -1
\end{array}\right] \text {. }
$$

Then Eq. (11) is equivalent to the following circuit:

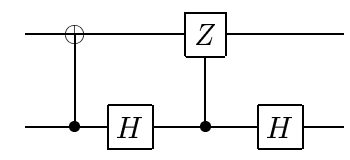

- Fact 3: A quantum-controlled gate can be replaced by a classically-controlled operation when the control qubit is measured.

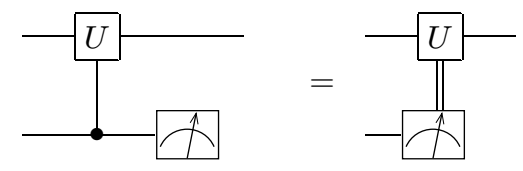

The meter represents the measurement of $Z$, which projects the measured state onto $|0\rangle$ or $|1\rangle$. The double line coming out of the meter carries the classical measurement result, and $U$ is performed if the measurement result is $|1\rangle$.

In Eq. (3), the two qubits are disentangled before the second Hadamard gate. Therefore, the second qubit can be measured before the second Hadamard gate without affecting the unknown state in the first qubit. Applying fact 3 to Eq. (3) results in the following circuit: 


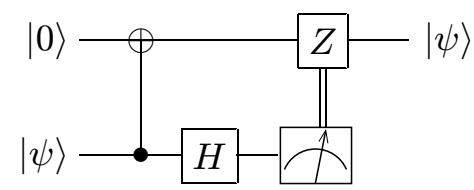

The circuit in Eq. (5) uses a CNOT and only one qubit for the ancilla. Therefore it is a one-bit teleportation circuit, which we refer to as " $Z$-teleportation" because a classically-controlled- $Z$ is applied after the measurement.

Using $Z$-teleportation we can derive other one-bit teleportation circuits. For instance, the following circuit first teleports the state $H|\psi\rangle$ using $Z$-teleportation, and then applies $H^{\dagger}=H$ to the teleported state $H|\psi\rangle$ to obtain the original state $|\psi\rangle$ :

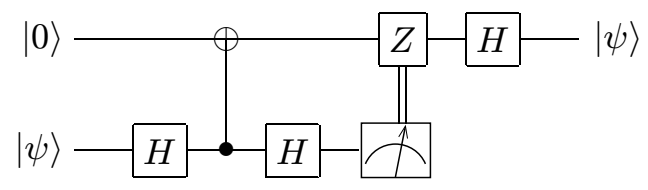

This circuit can be simplified to

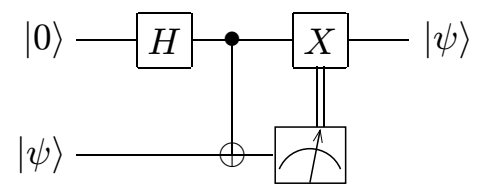

which we refer to as " $X$-teleportation". Similarly, we can derive other one-bit teleportation circuits as discussed in Appendix A. We will focus on $X$ and $Z$-teleportation circuits because they are sufficient for our construction in this paper.

$X$ and $Z$-teleportation circuits can both be represented using the same general structure:

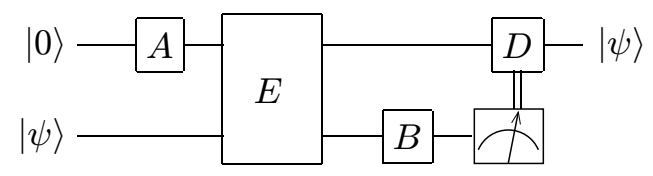

where the first qubit (the ancilla qubit) is initially in the $|0\rangle$ state. For $Z$-teleportation, $A=I$ ( $I$ is the $2 \times 2$ identity operator), $B=H, D=Z$, and $E$ is a CNOT with the first qubit as its target. For $X$-teleportation, $A=H, B=I, D=$ $X$, and $E$ is a CNOT with the first qubit as its control.

\section{FAULT-TOLERANT GATE CONSTRUCTION USING ONE-BIT TELEPORTATION}

In this section, we develop a general method for fault-tolerant gate construction using one-bit teleportation as a basic primitive. We will confine our attention to the Calderbank-Shor-Steane (CSS) codes that are doubly even and self-dual [13,14,1], although the results can be extended to any other stabilizer codes [9].

\section{A. Fault-tolerant gate hierarchy}

We first summarize the fault-tolerant gate hierarchy introduced in [9]. Let $C_{1}$ denote the Pauli group. Then for $k \geq 2$, we can recursively define $C_{k}$ as

$$
C_{k} \equiv\left\{U \mid U C_{1} U^{\dagger} \subseteq C_{k-1}\right\}
$$

For every $k, C_{k} \supset C_{k-1}$, and the set difference $C_{k} \backslash C_{k-1}$ is nonempty. For instance, $\operatorname{diag}\left(1, e^{i 2 \pi / 2^{k}}\right) \in C_{k} \backslash C_{k-1}$.

$C_{2}$ is a group called the Clifford group [8], which is the set of operators that conjugate Pauli operators into Pauli operators. Besides the Pauli operators, $C_{2}$ also contains other important gates, such as the CNOT, $H$, and the phase gate $S$ (defined by $S|x\rangle=i^{x}|x\rangle$ for $x \in\{0,1\}$ ). For doubly even and self-dual CSS codes, any encoded $C_{2}$ gate has transversal unitary implementation [1.5], which is fault-tolerant. 
$C_{2}$ gates alone, however, are not sufficient for universal quantum computation [8]. An additional gate outside $C_{2}$ is necessary and sufficient to complete universality [15]. In particular, adding any one of the following gates in $C_{3} \backslash C_{2}$ to the Clifford group results in a universal set of unitary operations: the $\pi / 8$ gate $T\left(T|x\rangle=e^{i \pi x / 4}|x\rangle\right.$ for $\left.x \in\{0,1\}\right)$, the controlled-phase gate $\Lambda_{1}(S)\left(\Lambda_{1}(S)|x y\rangle=i^{x \cdot y}|x y\rangle\right.$ for $\left.x, y=\{0,1\}\right)$, and the Toffoli gate (controlled-controlledNот) [1, 16, 6].

The construction of an encoded operation in $C_{3} \backslash C_{2}$ is much more complicated than that of an encoded operation in $C_{2}$, and requires quantum measurement and a particular ancilla state. But applying a $C_{3} \backslash C_{2}$ gate to certain known states can be replaced by direct preparation of the final states, which can be relatively easier as stated in the following: Theorem 1: Let $U$ be an $n$-qubit gate in $C_{3}$. Then the encoded state $U\left(|0\rangle^{\otimes n}\right)$ can be prepared fault-tolerantly by applying and measuring $C_{2}$ operators.

Proof: See Appendix B.

Since $C_{3}$ is closed under multiplication by elements in $C_{2}$ [19], Theorem 1 is also applicable when $|0\rangle^{\otimes n}$ is replaced by $V\left(|0\rangle^{\otimes n}\right)$ for $V \in C_{2}$, because $U|\psi\rangle=U V\left(|0\rangle^{\otimes n}\right)$ with $U V \in C_{3}$. We will use Theorem 1 in our fault-tolerant logic gate construction.

\section{B. $C_{3}$ gate construction using one-bit teleportation}

We now consider a general method of constructing fault-tolerant gates in $C_{3}$ using the one-bit teleportation scheme as a primitive. The basic idea is the following. To apply the encoded operation $U$ to an encoded state $|\psi\rangle$, we can first teleport $|\psi\rangle$ by either $X$ or $Z$-teleportation, and apply $U$ to the reconstructed $|\psi\rangle$. The extra teleportation step can be done fault-tolerantly because both $X$ and $Z$-teleportation use $C_{2}$ gates only. It further reduces the problem of fault-tolerant construction of a quantum logic gate to fault-tolerant preparation of a particular ancilla state. The reason for the reduction is that $U$ is applied to the ancilla, which is originally in the known state $|0\rangle$. If $U$ can be commuted backwards until it is applied to a known state without introducing more complicated gates, we can prepare the resulting known state, without applying $U$ directly, as the input ancilla. Using such an ancilla, the reconstructed state after the modified one-bit teleportation circuit will be $U|\psi\rangle$. That is, the encoded $U$ has been applied to the encoded $|\psi\rangle$ fault-tolerantly.

We now detail the formal construction. Let $U \in C_{3}$ be an $n$-qubit gate to be applied to $|\psi\rangle$, an encoded quantum state with $n$ logical qubits. We first teleport each logical qubit using either $X$ or $Z$-teleportation such that $|\psi\rangle$ is reconstructed in the ancilla, which is initially in the $|0\rangle^{\otimes n}$ state. We then apply $U$ to the reconstructed $|\psi\rangle$ to obtain $U|\psi\rangle$. This is described by the following quantum circuit:

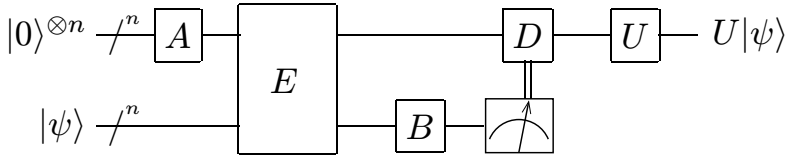

In Eq. (10), a register (wire) with the symbol "/ $n$ " represents a bundle of $n$ logical qubits. $A$ is a bitwise operation, $A=A_{1} \otimes \cdots \otimes A_{n}$, where $A_{i}$ acts on the $i^{\text {th }}$ logical qubit only. $B$ is a bitwise operation similar to $A$. $E$ is a tensor product such that $E=E_{i} \otimes \cdots \otimes E_{n}$, where each $E_{i}$ is a CNOT between the $i^{\text {th }}$ logical qubits of $|\psi\rangle$ and the known ancilla. The measurement box measures $Z$ bitwise and the double line represents the $n$-bit classical outcome. The $i^{t h}$ classical bit controls whether an operator $D_{i}$ is performed on the $i^{t h}$ logical state in the first register. This is denoted by $D$ for the sake of simplicity.

According to Sec. [I], if $Z$-teleportation is applied to the $i^{t h}$ logical qubit, $A_{i}=I, B_{i}=H, D_{i}=Z$, and $E_{i}$ is a CNOT with the first qubit as its target; if $X$-teleportation is applied instead, $A_{i}=H, B_{i}=I, D_{i}=X$, and $E_{i}$ is a CNOT with the first qubit as its control.

We now commute $U$ backwards in time. Commuting $U$ with the classically-controlled operation $D$ changes $D$ to $U D U^{\dagger}$. As $D \in C_{1}$ and $U \in C_{3}, U D U^{\dagger} \in C_{2}$ can still be performed transversally. Likewise, commuting $U$ with $E$ changes $E$ to $U E U^{\dagger}$ 10]. As CNOT $\notin C_{1}$, the resulting operation $U E U^{\dagger}$ may not be in $C_{2}$ for an arbitrary $U \in C_{3}$. To ensure $U E U^{\dagger} \in C_{2}$, we only consider $U$ that commutes with $E$ such that $U E U^{\dagger}=E \in C_{2}$. Then Eq. (10) becomes

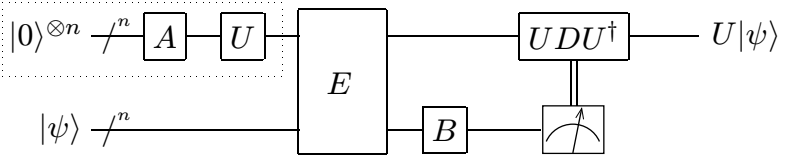


All the circuit elements outside the dotted box can be performed fault-tolerantly. Therefore, if we can prepare the input ancilla in the state $U A\left(|0\rangle^{\otimes n}\right)$, we can apply $U \in C_{3}$ to any encoded state $|\psi\rangle$ fault-tolerantly. As $A \in C_{2}, U A$ is also a $C_{3}$ operation. By Theorem 1 , the ancilla state $U A\left(|0\rangle^{\otimes n}\right)$ can be created fault-tolerantly. The stabilizers of such an ancilla state, which will be measured in preparing the state, can be easily derived. Recall that when $A_{i}=I$, $D_{i}=Z_{i}$, and when $A_{i}=H, D_{i}=X_{i}$. Therefore, $A_{i} Z_{i} A_{i}^{\dagger}=D_{i}$ is always true [18], and the stabilizers of $U A\left(|0\rangle^{\otimes n}\right)$ are $U A_{i} Z_{i} A_{i}^{\dagger} U^{\dagger}=U D_{i} U^{\dagger} \in C_{2}$.

Using the above method, we can systematically construct interesting gates in $C_{3} \backslash C_{2}$, including the $\pi / 8$, controlledphase, and Toffoli gate, as will be shown in Section IV.

Finally, we remark that the $C_{3}$ gates commuting with $E$ are not the only gates that can be performed by the one-bit teleportation scheme. Any $C_{3}$ gate of the form $U=G_{b} V G_{a}$ for $V$ commuting with $E$ and $G_{a}, G_{b} \in C_{2}$ can be performed using the generalized one-bit teleportation circuits. We discuss this in Appendix A.

\section{Recursive construction}

In this section, we extend our discussion to the gates in $C_{k}$ and characterize a class of gates that can be recursively constructed with one-bit teleportation as a basic primitive.

We prove by induction that the diagonal subset of $C_{k}$, defined by $F_{k}=\left\{U \in C_{k}\right.$ and $U$ is diagonal $\}$, can be recursively constructed.

First, when $U \in F_{k}$, we choose to apply $X$-teleportation to each logical qubit. In this case, each $E_{i}$ is a CNOT taking the $i^{t h}$ logical qubit in the ancilla as the control bit. Therefore, $E$ commutes with $U$ and Eq. (11) holds with $A_{i}=H, B_{i}=I$ and $D_{i}=X_{i}$ for $i=1, \ldots, n$. Second, since for $U \in F_{k}$ and $P \in C_{1}, U P U^{\dagger}=U P$ for some $\tilde{U} \in F_{k-1}$ [19], $U D_{i} U^{\dagger}=U X_{i} U^{\dagger}=U_{x} X_{i}$ for some $U_{x} \in F_{k-1}$. Therefore, if the gates in $F_{k-1}$ can be performed, the classically-controlled operation $U D_{i} U^{\dagger}$ for $U \in F_{k}$ can also be performed. Third, the required ancilla $U H^{\otimes n}\left(|0\rangle^{\otimes n}\right)$ can be prepared fault-tolerantly with recursive construction as shown in Appendix B. Finally, the gates in $F_{2} \subset C_{2}$ have transversal implementation. By induction, all the gates in $F_{k}$ can be performed fault-tolerantly with recursive application of the one-bit teleportation scheme.

The sets $F_{k}$ contain many interesting gates, such as $V^{k}=\operatorname{diag}\left(1, e^{i \pi / 2^{k}}\right)$, which are the single qubit $\pi / 2^{k}$ rotations, and $\Lambda_{1}\left(V^{k-1}\right)=\operatorname{diag}\left(1,1,1, e^{i \pi / 2^{k-1}}\right)$, which are the controlled rotations used in the quantum Fourier transform circuit [11,20] essential to Shor's factoring algorithm [11]. $F_{k}$ also includes the multiple-qubit gates $\Lambda_{n}\left(V^{l}\right)$ for $n+l \leq k$ [19], where $\Lambda_{n}\left(V^{l}\right)$ applies $V^{l}$ to the $(n+1)^{t h}$ qubit if and only if the first $n$ qubits are all in the state $|1\rangle$. By the closure property of $F_{k}$ [19], all products of $\Lambda_{n}\left(V^{l}\right)$ for $n+l \leq k$ are in $F_{k}$. To perform gates in $F_{k}$ for $k$ small, the recursive construction we have described can be more efficient than approximating these gates to the same accuracy using a universal set of fault-tolerant quantum logic gates.

The gates in $F_{k}$ are not the only ones that can be constructed using the one-bit teleportation scheme. For instance, if $U \in C_{k}$ is related to an element in $F_{k}$ by conjugation with Hadamard gates in the $i_{1}^{t h}, \ldots, i_{l}^{t h}$ qubits, $E$ can be made to commute with $U$ by applying $Z$ teleportation to the $i_{1}^{t h}, \ldots, i_{l}^{t h}$ qubits and $X$-teleportation to the rest. The Toffoli gate is an example. More generally, any gate that is a product of $C_{2}$ gates and a single $F_{k}$ gate can be constructed recursively.

\section{EXAMPLES}

In this section, we systematically construct three important fault-tolerant gates in $C_{3} \backslash C_{2}$ using the general method described in Sec. III. Any one of these gates, together with the Clifford group, forms a universal set of gates. For each of the construction, we will derive the required circuit and the ancilla. The ancilla can always be prepared fault-tolerantly (see Appendix B).

\section{A. The $\pi / 8$ gate}

The $\pi / 8$ gate, $T$, has the following matrix representation:

$$
T=\left[\begin{array}{cc}
1 & 0 \\
0 & e^{i \pi / 4}
\end{array}\right]
$$


As $T$ is diagonal, following the recipe in Sec. III, we choose to apply $X$-teleportation to $|\psi\rangle$ and apply $T$ to the teleported $|\psi\rangle$ :

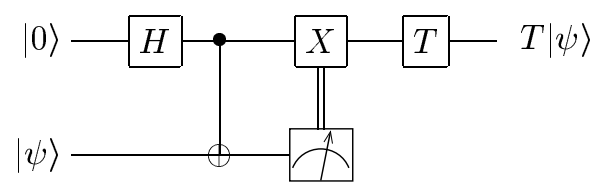

We commute $T$ backwards using two facts. First, $T X T^{\dagger}=e^{-i \pi / 4} S X$, where the phase gate $S$ is defined in Sec. III A. Second, $T$ commutes with the CNOT by construction. Thus, we obtain a circuit to implement the $\pi / 8$ gate (where an irrelevant overall phase has been ignored):

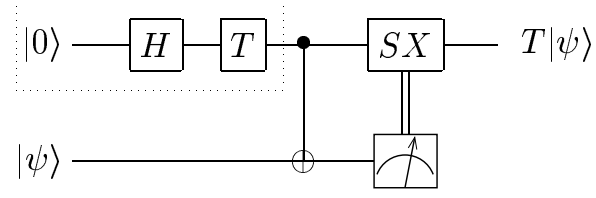

All the circuit elements outside the dotted box can be performed fault-tolerantly. The dotted box, then, can be replaced by an ancilla in the state

$$
\left|\phi_{+}\right\rangle=T H|0\rangle=\frac{|0\rangle+e^{i \pi / 4}|1\rangle}{\sqrt{2}},
$$

which can be prepared fault-tolerantly as described in Appendix B. Thus, we have derived a circuit and the corresponding ancilla for performing the fault-tolerant $\pi / 8$ gate. We note that this re-derives the same circuit and ancilla state used in [6.

\section{B. The controlled-phase gate}

The controlled-phase gate $\Lambda_{1}(S)$ (defined in Sec. [II A) is in $C_{3}$, and forms a universal set of gates [16, 12] together with $H$ and CNOT.

We use the following circuit symbol for $\Lambda_{1}(S)$ :

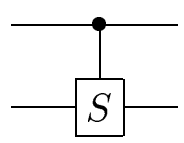

$\Lambda_{1}(S)$ commutes with $Z_{i}$, and conjugates $X_{i}(i=1,2)$ as follows:

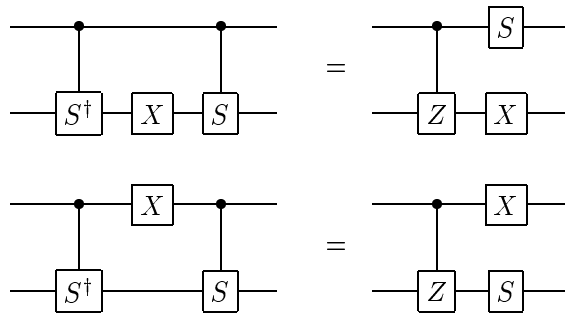

where the controlled- $Z$ operation, $\Lambda_{1}(Z)$, acts on basis states as $\Lambda_{1}(Z)|x\rangle|y\rangle=(-1)^{x \cdot y}|x\rangle|y\rangle$. To construct $\Lambda_{1}(S)$, we first teleport the two-qubit state $|\psi\rangle$ and apply $\Lambda_{1}(S)$. This linear transformation preserves phase coherence, and thus, it suffices to consider its action on the basis states $|x y\rangle$. Since $\Lambda_{1}(S)$ is diagonal, we apply $X$-teleportation to both qubit states such that the CNOTs in the circuit commute with $\Lambda_{1}(S)$.

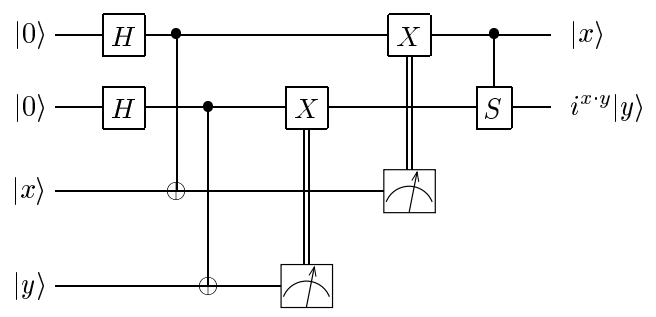


Commuting $\Lambda_{1}(S)$ backwards using the commutation rules in Eqs. (17)-(18), we obtain a circuit for the controlledphase gate:

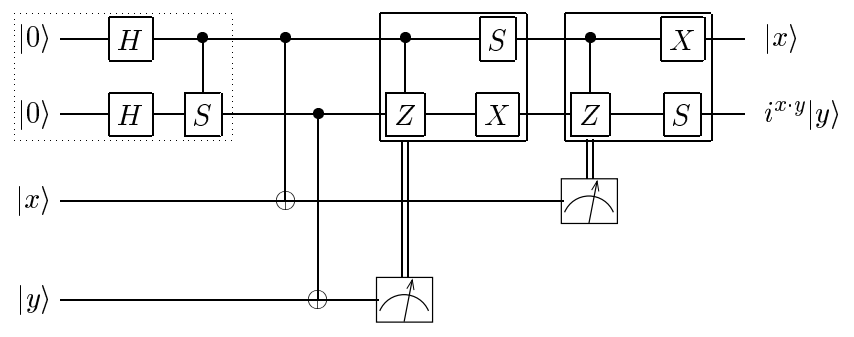

where the double lines control all the operations in the corresponding boxes. All the circuit elements in Eq. (20), except those in the dotted box, can be performed fault-tolerantly. Finally, we can replace the dotted box by an input ancilla in the following state:

$$
\begin{aligned}
\left|\phi_{+}\right\rangle & =\Lambda_{1}(S)\left(H_{1} \otimes H_{2}\right)|00\rangle \\
& =\frac{1}{2}(|00\rangle+|01\rangle+|10\rangle+i|11\rangle),
\end{aligned}
$$

which can be prepared fault-tolerantly. This completes the requirement for performing the controlled-phase gate fault-tolerantly.

\section{The Toffoli gate}

To construct the Toffoli gate (controlled-controlled-NOT), we begin with some useful commutation rules:

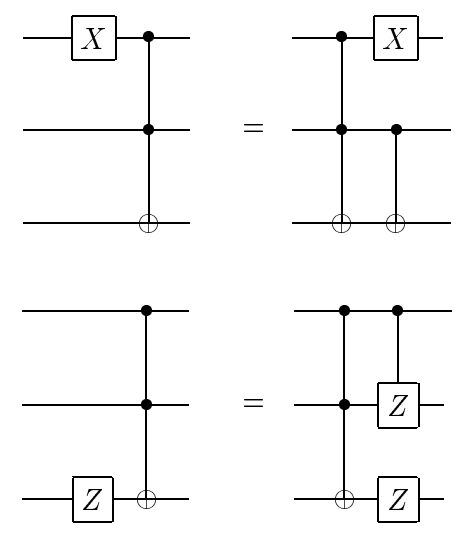

As in the controlled-phase gate construction, we demonstrate the construction on basis states $|x y z\rangle$ for three qubits. We first teleport $|x y z\rangle$ and then apply a Toffoli gate. Since the Toffoli gate is diagonalized by a Hadamard transform on the target qubit, the choice of $X$-teleportation for the control qubits and $Z$-teleportation for the target qubit ensures that the three CNOTs commute with the Toffoli gate.

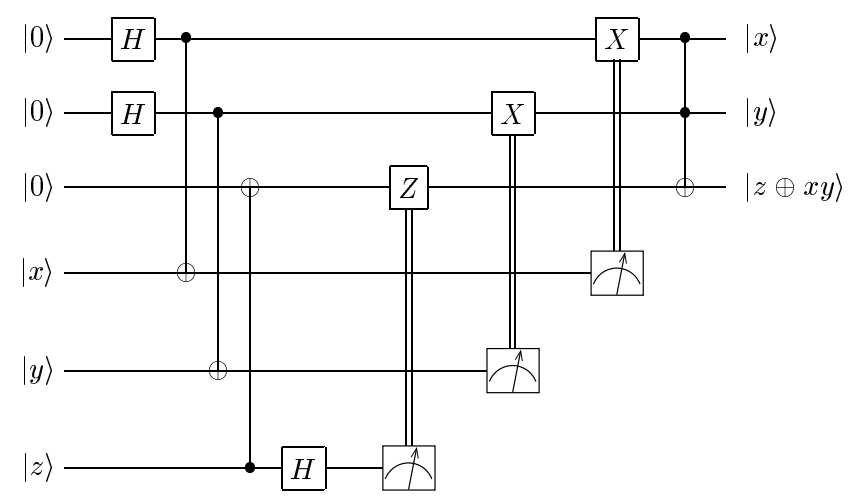


Commuting the Toffoli gate backwards to the far left using Eqs. (23)-(24), Eq. (25) is equivalent to

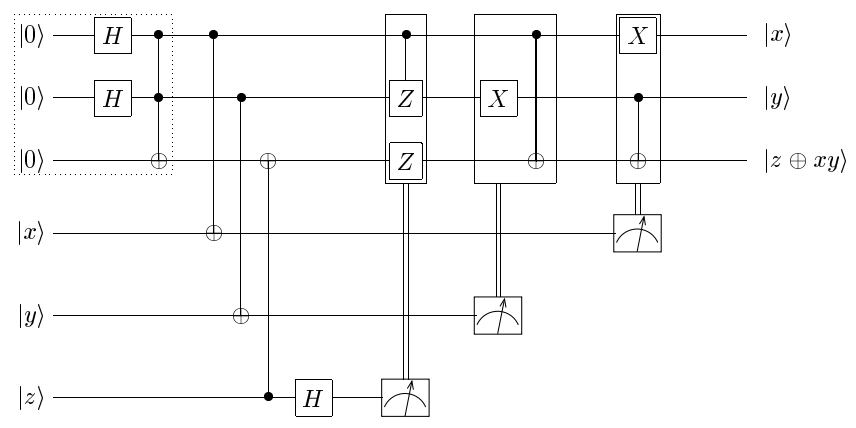

All the circuit elements except those in the dotted box can be performed fault-tolerantly. It remains to prepare the state created in the dotted box,

$$
\begin{aligned}
\left|\phi_{+}\right\rangle & =U\left(H_{1} \otimes H_{2}\right)|000\rangle \\
& =\frac{1}{2}(|000\rangle+|010\rangle+|100\rangle+|111\rangle),
\end{aligned}
$$

where $U$ denotes the Toffoli gate. Again this ancilla state can be prepared fault-tolerantly, as described in Appendix B.

The ancilla and the quantum circuit derived here are the same as those in Shor's original construction [1]. The one-bit teleportation scheme elucidates the choice of the ancilla state and the procedure in [1].

\section{REMOTE GATE CONSTRUCTION USING ONE-BIT TELEPORTATION}

The one-bit teleportation scheme, in addition to being useful for fault-tolerant gate construction, can also be used to design a variety of remote quantum operations. Constructing remote quantum operations is related to constructing fault-tolerant gates in that both require a particular ancilla state to replace a prohibited operation. In this section, we use one-bit teleportation as a basic primitive to derive the quantum circuits and the required ancilla states for the two-bit quantum teleportation and the remote CNOT.

\section{A. Two-bit teleportation}

Suppose Alice needs to send a qubit state $|\psi\rangle$ to Bob. Direct quantum communication is not allowed, but Alice and Bob can share some ancilla state. The question is, how can Alice send $|\psi\rangle$ to Bob? A well-known solution to this problem is quantum teleportation [7], which uses an EPR state and classical communication. Using one-bit teleportation, we give an alternative derivation of the required (entangled) ancilla and the required teleportation circuit.

We first construct a circuit to send the unknown state with a prohibited operation. Then we remove the requirement of such a prohibited operation. Let $|\psi\rangle$ be the state to be communicated from Alice to Bob. Alice can send $|\psi\rangle$ to Bob by applying one-bit teleportation twice. Step 1: Alice swaps $|\psi\rangle$ with an ancilla $|0\rangle$ using $X$-teleportation. Step 2: Alice sends the teleported $|\psi\rangle$ to Bob using $Z$-teleportation (with a prohibited CNOT in this step). The circuit representation for the process is

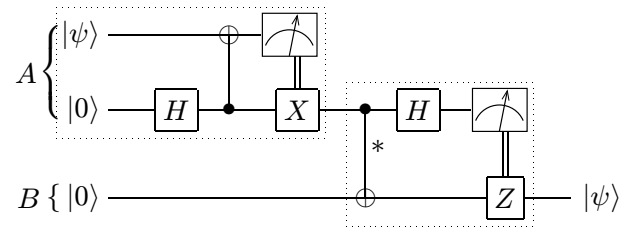

The prohibited operation (CNOT), which is marked by an asterisk, can be commuted backwards using the commutation relation: 


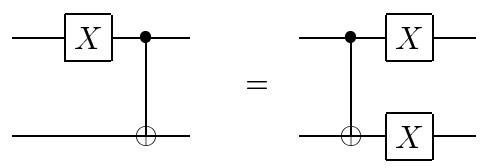

This leads to the usual quantum teleportation circuit

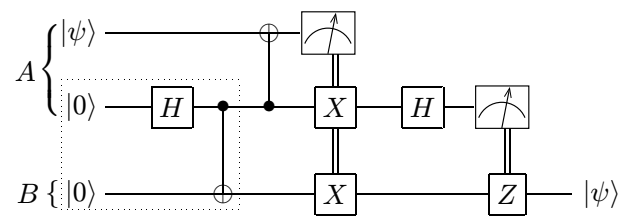

In Eq. (31), the prohibited CNOT acts on the known state inside the dotted box, which can be replaced by the following state it creates:

$$
|\phi\rangle=\Lambda_{1}(X) H_{1}|00\rangle=\frac{1}{\sqrt{2}}(|00\rangle+|11\rangle)
$$

In other words, if Alice shares this entangled state with Bob, the state $|\psi\rangle$ can be sent to Bob without quantum communication.

Note that the classically-controlled- $X$ on the second register only affects its overall sign, and can be omitted since the second register is subsequently measured.

An alternative circuit, which accomplishes the same task, can be derived when $Z$ and $X$-teleportation are used for the two steps instead. We start with the following circuit:

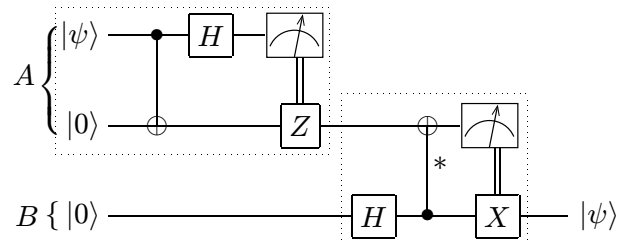

Using the commutation rule

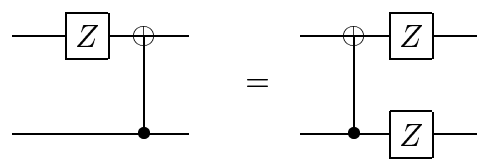

we can commute the prohibited CNOT backwards to obtain an equivalent quantum teleportation circuit

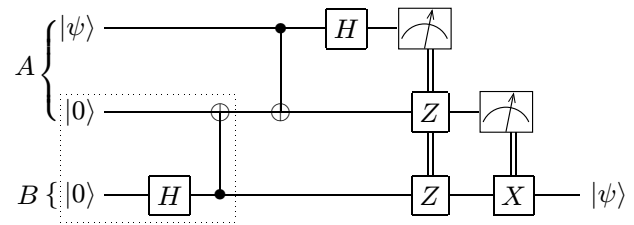

The disallowed element in the dotted box can be replaced by the EPR state of Eq. (32). The irrelevant classicallycontrolled- $Z$ on the second register can be omitted.

The two-bit teleportation circuits of Eqs. (31) and (35) are equivalent to that in [21], but as mentioned above, they are derived differently.

\section{B. Remote CNOT}

Suppose Alice and Bob have in their possession quantum states $|\alpha\rangle$ and $|\beta\rangle$, respectively. How can they perform a simple distributed computation, a CNOT from $|\alpha\rangle$ to $|\beta\rangle$, without communicating any quantum information between them, but perhaps with the aid of some initially shared standard quantum state? A solution to this problem is given 
in [8]. The ad-hoc method employed, however, does not suggest a systematic technique for deriving the solution, or solutions to generalized versions of this problem. Here, we use one-bit teleportation to present a general technique and derive a different circuit that accomplishes the same task.

Alice and Bob first swap their states with their respective ancilla state $|0\rangle$ by one-bit teleportation, and then apply a prohibited СNOT. The quantum circuit is chosen so that Alice uses $X$-teleportation and Bob uses $Z$-teleportation:

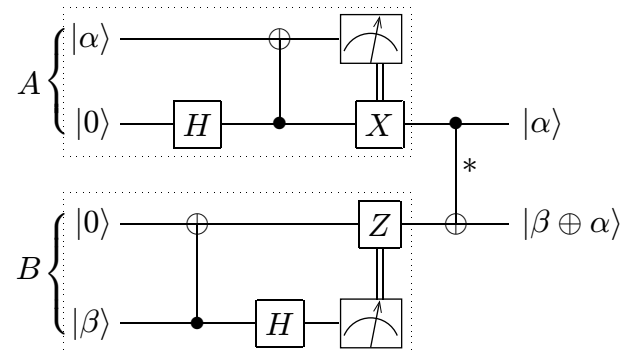

The prohibited CNOT can be commuted backwards to obtain a remote CNOT circuit:

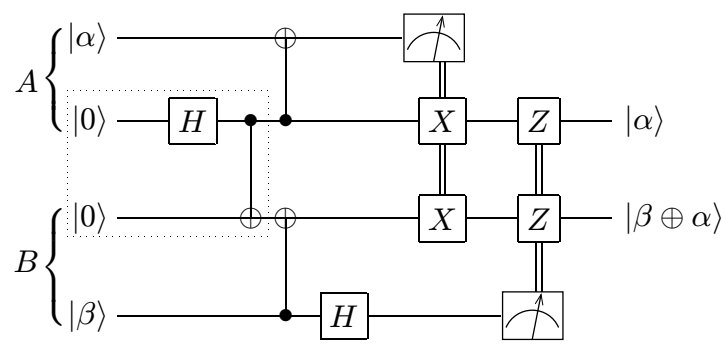

The prohibited operation in the dotted box is applied to a known state, and can be replaced by the EPR state of Eq. (32). Provided such a shared entangled state is initially available to Alice and Bob, they can perform a remote CNOT operation using two bits of classical communication.

Note that a remote CNOT can also be constructed by using two-bit teleportation twice in an obvious way: Bob first sends his qubit $|\beta\rangle$ to Alice with two-bit teleportation, and then Alice applies CNOT to $|\alpha\rangle|\beta\rangle$ and sends the qubit $|\alpha \oplus \beta\rangle$ to Bob with two-bit teleportation. Such construction, however, requires two pairs of maximally entangled state and four bits of classical communication, which is twice that required for the one-bit teleportation scheme.

Our remote CNOT construction in Eq. (37) is different from that in [8], which can also be derived using the one-bit teleportation scheme, as described in Appendix $\mathrm{G}$.

Finally, we remark that the two examples of constructing remote operations strengthen the concept of teleporting quantum logic gates with one-bit teleportation, as we have shown that if the input ancilla is a special state related to the CNOT gate, the reconstructed state is the one to which a CNOT gate has been applied.

\section{CONCLUSION}

We have presented a systematic technique to construct a variety of quantum operations, by using a primitive one-bit teleportation scheme. Such a scheme reduces the problem of constructing a quantum logic gate to preparing an ancilla state created by the same gate applied to a known state. The usefulness of this technique is particularly manifest for two kinds of application: fault-tolerant quantum computation and remote quantum computation, as demonstrated in our construction of the $\pi / 8$, controlled-phase, and Toffoli gates, and the remote-CNOT. With recursive application of the one-bit teleportation scheme, we can also construct an infinite hierarchy of gates fault-tolerantly.

The idea of teleporting quantum logic gates has been used in [9], with two-bit teleportation as a primitive, to perform universal quantum computation. The two-bit teleportation scheme allows all $C_{3}$ gates to be teleported fault-tolerantly, and all $C_{k}$ gates to be teleported with recursive application of the scheme. For one-bit teleportation, however, we can only provide sufficient conditions for gates in $C_{3}$ to be teleportable, namely, any $C_{3}$ gate that can be written as a product of $C_{2}$ gates and a single $C_{3}$ gate that commutes with CNOT. It is not known if this includes all the $C_{3}$ gates. The difficulty in describing the exact set of one-bit teleportable $C_{3}$ gates arises from the requirement for a $C_{2} \backslash C_{1}$ gate in the one-bit teleportation circuit. Such a $C_{2} \backslash C_{1}$ gate may be conjugated outside $C_{2}$ by a $C_{3}$ gate, and therefore cannot be directly performed fault-tolerantly. This places further constraint on the teleportable $U \in C_{k}$ for 
$k>3$. Because of our present lack of understanding of the general structure and nature of $C_{k}$ gates, the distinction between the ultimate capabilities of the one and two-bit teleportation schemes remains an interesting and difficult open question.

Nevertheless, as we have shown, one-bit teleportation can provide much simpler protocols than two-bit teleportation in constructing quantum logic gates. This is because one-bit teleportation only requires projective measurement of $Z$ and as many ancilla qubits as the state to be transformed; two-bit teleportation, however, requires Bell measurement and twice as many ancilla qubits as the original state.

At a very general level, the logical gate teleportation schemes reduce the difficulty of constructing quantum logic gates by using special ancilla states. This can be useful not only for simplifying hardware requirements, but also for designing and optimizing computation and communication protocols 22,23]. Even more intriguing, perhaps, is that this result gives us a first glimpse at what might someday be a standard architecture for a quantum computer: a simple assembly of one-bit teleportation primitives, capable of universal quantum computation on quantum data, given the assistance of standard quantum states that are obtained as commercial resources. The definition of such a stored-program architecture could be pivotal in the development of this field, much as the von Neumann or Harvard architecture 24] were important in classical computation.

\section{ACKNOWLEDGMENTS}

The relation between fault-tolerant quantum logic gate construction and teleportation is first alluded to by Shor [i]. The $X$ and $Z$ teleportation circuits presented in this paper are due to Charles Bennett and Daniel Gottesman (unpublished). We are grateful to Daniel Gottesman for introducing us to the interesting subject of the $C_{k}$ hierarchy, and for enlightening discussions. We thank Professor James Harris and Yoshihisa Yamamoto for support and encouragement. This work was supported by the DARPA Ultra-scale Program under the NMRQC initiative, contract DAAG55-97-1-0341, administered by the Army Research Office. D.L. acknowledges support from the IBM Fellowship program and Nippon Telegraph and Telephone Corporation (NTT).

\section{APPENDIX A: GENERALIZATIONS OF THE ONE-BIT TELEPORTATION CIRCUITS}

The one-bit teleportation circuit used in fault-tolerant gate construction has three components: a particular input ancilla, a sequence of $C_{2}$ gates, and finally the measurement and classically-controlled operation. The teleportability of one-bit teleportation is governed by the sequence of $C_{2}$ gates before the measurement. Using the $X$ and $Z$ teleportation circuits of Eq. (11), any $U \in C_{3}$ that commutes with $E$ can be teleported. In this appendix, we derive other one-bit teleportation circuits, which use different $C_{2}$ gates, and then discuss their application in constructing fault-tolerant gates.

By teleporting $G|\psi\rangle$ using $X$-teleportation and applying $G^{\dagger}$ to the teleported $G|\psi\rangle$, we obtain the following generalized one-bit telelportation circuit:

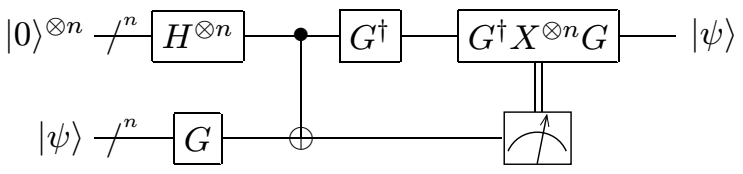

When $G=I^{\otimes n}$ and $H^{\otimes n}$, Eq. (A1) reduces to the $X$ and $Z$-teleportation circuits.

In Sec. [II], we showed that all the operations in $F_{3}$ can be performed fault-tolerantly using $X$-teleportation. Here, we generalize the result to show that, if $U \in C_{3}$ and $U=G_{b} V G_{a}$, where $V \in F_{3}$ and $G_{a}, G_{b} \in C_{2}$, then $U$ can be performed fault-tolerantly using the general one-bit teleportation scheme by the following procedure:

Step 1: Using the circuit of Eq. (A1) with $G=G_{a}$, we first teleport the state $|\psi\rangle$ to the ancilla initialized in the state $|0\rangle^{\otimes n}$, and then apply $U$ to the ancilla. This can be represented by

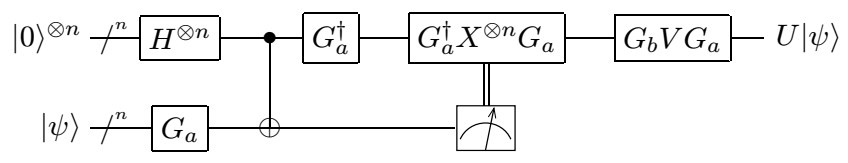

Step 2: Commuting $U$ backwards, one obtains 


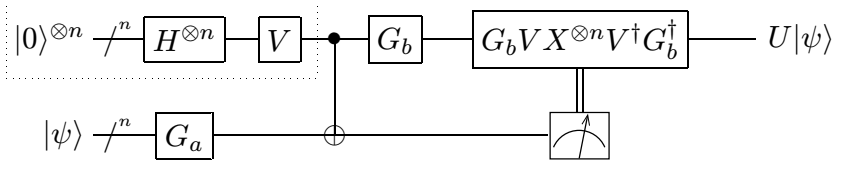

Note that the new classically-controlled operation is $G_{b} V X^{\otimes n} V^{\dagger} G_{b}^{\dagger}$, which is in $C_{2}$ because $V X^{\otimes n} V^{\dagger} \in C_{2}$. Therefore, all the circuit elements can be performed fault-tolerantly, except those in the dotted box, which can be replaced by an ancilla in the state $V H^{\otimes n}\left(|0\rangle^{\otimes n}\right)$.

There are $C_{3}$ gates that cannot be constructed using $X$ and $Z$-teleportation directly, but can be constructed using other one-bit teleportation circuits. For instance, the controlled-Hadamard gate $\Lambda_{1}\left(H_{2}\right) \in C_{3} \backslash C_{2}$ does not commute with $E$ in Eq. (10) for all possible combinations of $X$ and $Z$-teleportation circuits, but $\Lambda_{1}\left(H_{2}\right)$ can be written as $G_{b} V G_{a}$ with $G_{a}=Q_{2}^{\dagger}, G_{b}=\Lambda_{1}\left(X_{2}\right) Q_{2}$ and $V=T_{1} \Lambda_{1}\left(S_{2}^{\dagger}\right)$, where $Q=S^{\dagger} H S \in C_{2}$. Thus, $\Lambda_{1}\left(H_{2}\right)$ can still be performed using the general one-bit teleportation scheme.

We remark that a $C_{3}$ gate $U=G_{b} V G_{a}$ with $G_{a}, G_{b} \in C_{2}$ and $V \in F_{3}$ can be performed indirectly by applying $G_{a}, V$ and $G_{b}$ in sequence, where $V$ is applied by $X$-teleportation. If the operations in the generalized one-bit teleportation circuit, $G_{b}, \Lambda_{1}(X)$, and $G_{a}$ of Eq. (A3), are also considered, the total requirements to perform $U$ by such indirect implementation and by direct one-bit teleportation are almost the same. But if we are given different one-bit teleportation circuits as primitives, we can use them to directly teleport different sets of $C_{3}$ gates. In other words, if we are given the circuit of Eq. (11), using an input ancilla in the state $U A\left(|0\rangle^{\otimes n}\right)$, we can teleport $U \in C_{3}$ that commutes with $E$; if we are given the circuit of Eq. (A3), using an input ancilla in the state $V H^{\otimes n}\left(|0\rangle^{\otimes n}\right)$, we can teleport $U$ in the form of $G_{b} V G_{a}$. In this sense, then, the generalized one-bit teleportation circuits are interesting and allow more gates in $C_{3}$ to be teleported directly.

\section{APPENDIX B: FAULT-TOLERANT STATE PREPARATION}

In this section, we first prove Theorem 1 in Sec. III A by construction. We then show how to create the three ancilla states in Sec. IV] fault-tolerantly. Finally, we explain how to prepare a class of encoded quantum states fault-tolerantly by recursive application of the one-bit teleportation scheme.

\section{Fault-tolerant preparation of quantum states}

A stabilizer of a quantum state is a quantum operator that transforms the state to itself. Let $\mathcal{C}$ be the codeword space corresponding to an $[[m, n]]$ stabilizer code, which encodes $n$ logical qubits using $m$ physical qubits. The stabilizer $S$ of $\mathcal{C}$ is an Abelian subgroup of the Pauli group, or $C_{1}$, such that $|\psi\rangle \in \mathcal{C}$ if and only if $\forall M \in S, M|\psi\rangle=|\psi\rangle$. By performing error correction for the stabilizer code, we can project an arbitrary state onto an encoded state in $\mathcal{C}$ [8],2].

The stabilizer $S$ has $2^{m-n}$ elements generated by $m-n$ independent operators in $C_{1}$, and defines a quantum code of dimension $2^{n}$. Each encoded state is, then, determined by $n$ extra independent stabilizers. (In the following, we will restrict our discussion to the codeword space and exclude the stabilizers of the code from the stabilizers of an encoded state.) For instance, the encoded $|0\rangle^{\otimes n}$ is determined by $Z_{i}$ for $i=1, \ldots, n$, where $Z_{i}$ is the encoded $Z$ on each logic qubit. In general, stabilizers need not commute with one another and need not square to the identity. But an independent set of stabilizers can always be chosen to be a mutually commuting set of elements that square to the identity. This is because $|\psi\rangle=U\left(|0\rangle^{\otimes n}\right)$ for some encoded $U$, leading to a possible choice $\mathrm{St}(|\psi\rangle) \equiv\left\{U Z_{i} U^{\dagger}, i=1, \ldots, n\right\}$ with the desired properties. We restate the above as a lemma:

Lemma 1: For any $|\psi\rangle, \operatorname{St}(|\psi\rangle)$ can be chosen such that $\forall M, N \in \operatorname{St}(|\psi\rangle)$, (a) $M^{2}=I$ and (b) $[M, N]=M N-N M=$ 0 .

Note that the elements in $\operatorname{St}(|\psi\rangle)$ are all valid encoded operations, and their actions preserve the codeword space.

As a quantum state is the simultaneous +1 eigenstate of its stabilizers, the state can be prepared by projecting an arbitrary encoded state onto the simultaneous +1 eigenstate of its stabilizers. In the following we will show how to create a class of quantum states fault-tolerantly by measuring their stabilizers.

Given a quantum state $|\psi\rangle$ encoded with an $[[m, n]]$ stabilizer code, the operator $M \in C_{2}$ with $M^{2}=I$ can be measured fault-tolerantly on $|\psi\rangle$ as follows. First, we prepare a cat state

$$
\mid \text { cat }\rangle \equiv \frac{1}{\sqrt{2}}(|\overline{0}\rangle+|\overline{1}\rangle)
$$


where $|\bar{i}\rangle$ consists of $m$ physical qubits in the state $|i\rangle(i=0,1)$. (The cat state cannot be created fault-tolerantly, but it can always be verified [1].) For the doubly even and self-dual CSS codes, the encoded $M \in C_{2}$ can be written as $M=M^{1} \otimes \cdots \otimes M^{m}$, where $M^{j}$ acts only on the $j^{t h}$ physical qubit of each block of the encoded state $|\psi\rangle$. For each $j$, we perform controlled- $M^{j}$ with the $j^{\text {th }}$ qubit of the cat state as the control bit and the $j^{\text {th }}$ qubit of $|\psi\rangle$ as the target qubit. Effectively, a cat-state-controlled- $M$ is applied to the state $\mid$ cat $\rangle|\psi\rangle$ with transversal operations leading to the state

$$
\begin{aligned}
& \frac{1}{\sqrt{2}}|\overline{0}\rangle|\psi\rangle+\frac{1}{\sqrt{2}}|\overline{1}\rangle M|\psi\rangle \\
= & \frac{1}{2}(|\overline{0}\rangle+|\overline{1}\rangle)(I+M)|\psi\rangle+\frac{1}{2}(|\overline{0}\rangle-|\overline{1}\rangle)(I-M)|\psi\rangle .
\end{aligned}
$$

Note that as $M^{2}=I,(I \pm M)|\psi\rangle$ are \pm 1 eigenstates of $M$ for any $|\psi\rangle$.

We can measure the cat state fault-tolerantly using the procedure described in [1, 2] to distinguish $|\overline{0}\rangle+|\overline{1}\rangle$ from $|\overline{0}\rangle-|\overline{1}\rangle$. (We omit the unimportant normalization factors.) If we obtain $|\overline{0}\rangle+|\overline{1}\rangle$, the encoded state is projected onto $(I+M)|\psi\rangle$, the +1 eigenstate of $M$; otherwise the resulting encoded state is $(I-M)|\psi\rangle$, the -1 eigenstate of $M$, which may be transformed to a +1 eigenstate of $M$ by the following Lemma.

Lemma 2: If $M \in C_{2}, M^{2}=I$, and there exists $Q \in C_{2}$ such that $\{M, Q\}=M Q+Q M=0$, then we can always transform an arbitrary encoded state $|\psi\rangle$ onto a +1 eigenstate of $M$ using fault-tolerant operations. The resulting +1 eigenstate is either $(I+M)|\psi\rangle$ or $(I+M) Q|\psi\rangle$, which can be written jointly as $(I+M) Q^{a}|\psi\rangle$ for $a=0$ or 1 .

Proof: We have shown that we can project an arbitrary encoded state $|\psi\rangle$ onto $(I \pm M)|\psi\rangle$, the \pm 1 eigenstate of $M$. If the resulting state is $(I+M)|\psi\rangle$, we are done; otherwise, we apply $Q \in C_{2}$ fault-tolerantly to $(I-M)|\psi\rangle$. Since $Q$ anticommutes with $M$, it transforms the -1 eigenstate of $M$ to a +1 eigenstate of $M$ as follows: $Q(I-M)|\psi\rangle=$ $(I+M)(Q|\psi\rangle)$. Thus, we can always obtain a +1 eigenstate of $M$, which is $(I+M) Q^{a}|\psi\rangle$ for $a=0$ or 1 .

Next we will show that a special class of quantum states can be created fault-tolerantly.

Lemma 3: If $\operatorname{St}(|\psi\rangle)=\left\{M_{1}, \ldots, M_{n}\right\} \subset C_{2}$ and $\forall M_{i} \in \operatorname{St}(|\psi\rangle)$ there exists $Q_{i} \in C_{2}$ such that $\left\{M_{i}, Q_{i}\right\}=0$, and $\left[M_{i}, Q_{j}\right]=0$ for $i \neq j$, then $|\psi\rangle$ can be created fault-tolerantly by measuring the elements in $\operatorname{St}(|\psi\rangle)$ fault-tolerantly. Proof: By Lemma 1, $\forall i, M_{i}^{2}=I$. Starting from any encoded state $|\phi\rangle$, we measure $M_{1}, \ldots, M_{n}$ sequentially, and after each measurement we apply the corresponding operation $Q_{i}$ if the projected state is the -1 eigenstate of $M_{i}$. By Lemma 2, the resulting state is

$$
\begin{aligned}
|\psi\rangle & =\left(I+M_{n}\right) Q_{n}^{a_{n}} \cdots\left(I+M_{1}\right) Q_{1}^{a_{1}}|\phi\rangle \\
& =\left(I+M_{n}\right) \cdots\left(I+M_{1}\right) Q_{n}^{a_{n}} \cdots Q_{1}^{a_{1}}|\phi\rangle,
\end{aligned}
$$

where $a_{i}=0$ or 1 , and we have used the fact that $\left[M_{i}, Q_{j}\right]=0$ for $i \neq j$. As $\left[M_{i}, M_{j}\right]=0$, it is easily verified that $\forall i, M_{i}|\psi\rangle=|\psi\rangle$, and $|\psi\rangle$ is the desired state that has been created fault-tolerantly.

Theorem 1 in Sec IIIA immediately follows:

Theorem 1: $\forall U \in C_{3}, U$ can be applied to the encoded $|0\rangle^{\otimes n}$ state using $C_{2}$ operators and fault-tolerant measurement of $C_{2}$ operators.

Proof: Applying $U \in C_{3}$ to the encoded $|0\rangle^{\otimes n}$ state is equivalent to preparing the state $|\psi\rangle=U\left(|0\rangle^{\otimes n}\right)$, which has stabilizers $M_{i}=U Z_{i} U^{\dagger}$ for $i=1, \ldots, n$. Define $Q_{i} \equiv U X_{i} U^{\dagger} \in C_{2}$ for each $i$. Then $\left\{Z_{i}, X_{i}\right\}=0$ implies $\left\{M_{i}, Q_{i}\right\}=U\left\{Z_{i}, X_{i}\right\} U^{\dagger}=0$, and for $i \neq j,\left[Z_{i}, X_{j}\right]=0$ implies $\left[M_{i}, Q_{j}\right]=U\left[Z_{i}, X_{j}\right] U^{\dagger}=0$. Thus by Lemma 3 , the state $|\psi\rangle$ can be created fault-tolerantly.

\section{Examples}

To prepare a specific encoded state from an unknown encoded state we need to measure all its independent stabilizers. When the initial state is a known encoded state related to the desired state, we may not have to measure all the independent stabilizers. For instance, given two encoded states $|\phi\rangle$ and $\left|\phi^{\prime}\right\rangle$ with $\operatorname{St}(|\phi\rangle)=\left\{M_{1}, \ldots, M_{k}, M_{k+1}, \ldots, M_{n}\right\}$ and $\operatorname{St}\left(\left|\phi^{\prime}\right\rangle\right)=\left\{M_{1}, \ldots, M_{k}, M_{k+1}^{\prime}, \ldots, M_{n}^{\prime}\right\}$, the following state

$$
\left(I+M_{n}\right) \cdots\left(I+M_{k+1}\right) Q_{n}^{a_{n}} \cdots Q_{k+1}^{a_{k+1}}\left|\phi^{\prime}\right\rangle
$$

is the simultaneous +1 eigenstate of $M_{i}$ for $i=1, \ldots, n$. Thus, starting from $\left|\phi^{\prime}\right\rangle$, we can prepare the encoded state $|\phi\rangle$ by measuring only the $n-k$ different stabilizers. In the following, we will construct an initial state, with which, the desired state can be obtained by measuring only a single stabilizer. 
Assume we want to prepare the encoded state $\left|\psi_{+}\right\rangle=U\left(|0\rangle^{\otimes n}\right)$ for $U \in C_{3}$. Define $M_{i}$ and $Q_{i}$ for $i=1, \ldots, n$ as in the proof of Theorem 1. Then $Q_{i}\left|\psi_{+}\right\rangle$is a -1 eigenstate of $M_{i}$ such that $\left\langle\psi_{+}\left|Q_{i}\right| \psi_{+}\right\rangle=0$, and the following state

$$
|\psi\rangle=\frac{1}{\sqrt{2}}\left(\left|\psi_{+}\right\rangle+Q_{i}\left|\psi_{+}\right\rangle\right)
$$

is different from $\left|\psi_{+}\right\rangle$by only one independent stabilizer: $Q_{i}$ has replaced $M_{i}$. Therefore, the state $|\psi\rangle$ also satisfies the conditions of Lemma 3, and can be prepared fault-tolerantly. It follows that to obtain the state $\left|\psi_{+}\right\rangle$, we only need to measure the single stabilizer $M_{i}$ on $|\psi\rangle$.

To prepare an encoded state $\left|\psi_{+}\right\rangle$by preparing $|\psi\rangle$ first can be simpler than directly preparing $\left|\psi_{+}\right\rangle$from an arbitrary encoded state if $|\psi\rangle$ itself can be prepared easily. For instance, when $|\psi\rangle$ is a product state, it can be prepared by measuring only single qubit operators. We will describe how to prepare the required ancilla states for the three gates in Sec. IV. When the required ancilla $\left|\psi_{+}\right\rangle$is an entangled state with multiple-qubit stabilizers, we will construct it by preparing an easier state $|\psi\rangle$ first.

\section{a. Fault-tolerant preparation of the ancilla required for $T$ gate}

The required ancilla for constructing the $\pi / 8$ gate, $T$, is

$$
\left|\psi_{+}\right\rangle=T H|0\rangle=\frac{|0\rangle+e^{i \pi / 4}|1\rangle}{\sqrt{2}},
$$

with stabilizer

$$
M=(T H) Z(T H)^{\dagger}=e^{-i \pi / 4} S X,
$$

which anticommutes with $(T H) X(T H)^{\dagger}=Z$. Then starting from any encoded state, we can measure $M$, and apply $Z$ if the projected state is the -1 eigenstate, to create the state $\left|\psi_{+}\right\rangle$fault-tolerantly.

\section{b. Fault-tolerant preparation of the ancilla required for controlled-phase gate}

The required ancilla for constructing the controlled phase gate is

$$
\begin{aligned}
\left|\psi_{+}\right\rangle & =\Lambda_{1}(S)\left(H_{1} \otimes H_{2}\right)|00\rangle \\
& =\frac{1}{2}(|00\rangle+|01\rangle+|10\rangle+i|11\rangle),
\end{aligned}
$$

with stabilizers $M_{i}=\Lambda_{1}(S)\left(H_{1} \otimes H_{2}\right) Z_{i}\left(H_{1} \otimes H_{2}\right) \Lambda_{1}\left(S^{\dagger}\right)$ for $i=1,2$. Using Eqs. (17)-(18),

$$
\begin{aligned}
& M_{1}=\left(X_{1} \otimes S_{2}\right) \Lambda(Z), \\
& M_{2}=\left(S_{1} \otimes X_{2}\right) \Lambda(Z) .
\end{aligned}
$$

The corresponding operator that anticommutes with $M_{i}$ is $Q_{i}=\Lambda_{1}(S)\left(H_{1} \otimes H_{2}\right) X_{i}\left(H_{1} \otimes H_{2}\right) \Lambda_{1}\left(S^{\dagger}\right)=Z_{i}$ for $i=1,2$. $\left|\psi_{+}\right\rangle$is an entangled state, and both of $M_{1}$ and $M_{2}$ are two-qubit operators. But the following state

$$
\begin{aligned}
|\psi\rangle & =\frac{1}{\sqrt{2}}\left(\left|\psi_{+}\right\rangle+Q_{1}\left|\psi_{+}\right\rangle\right) \\
& =\frac{1}{\sqrt{2}}|0\rangle(|0\rangle+|1\rangle)
\end{aligned}
$$

is a product of single qubit states and has stabilizers $Z_{1}$ and $X_{2}$. Thus, we can first prepare $|\psi\rangle$ fault-tolerantly by measuring $Z_{1}$ and $X_{2}$, and then measure $M_{1}$ alone to get the state $\left|\psi_{+}\right\rangle$. Equivalently, we can also first prepare the state $\frac{1}{\sqrt{2}}\left(\left|\psi_{+}\right\rangle+Q_{2}\left|\psi_{+}\right\rangle\right)=\frac{1}{\sqrt{2}}(|0\rangle+|1\rangle)|0\rangle$, which has stabilizers $X_{2}$ and $Z_{1}$, and measure $M_{2}$ to obtain the state $\left|\psi_{+}\right\rangle$. 
The required ancilla for the Toffoli gate construction is

$$
\begin{aligned}
\left|\psi_{+}\right\rangle & =U\left(H_{1} \otimes H_{2}\right)|000\rangle \\
& =\frac{1}{2}(|000\rangle+|010\rangle+|100\rangle+|111\rangle),
\end{aligned}
$$

where $U$ is the Toffoli gate. The stabilizer of this state is $M_{i}=U\left(H_{1} \otimes H_{2}\right) Z_{i}\left(H_{1} \otimes H_{2}\right) U^{\dagger}$ for $i=1,2$, and 3 . Using Eqs. (23)-(24),

$$
\begin{aligned}
& M_{1}=X_{1} \otimes \mathrm{CNOT}_{23}, \\
& M_{2}=X_{2} \otimes \mathrm{CNOT}_{13}, \\
& M_{3}=Z_{3} \otimes \mathrm{CZ}_{12},
\end{aligned}
$$

where CZ represents a controlled- $Z$, and the ordered subscripts for CNOT and CZ specifies the control and target bits. The operator that anticommutes with $M_{i}$ is $Q_{i}=U\left(H_{1} \otimes H_{2}\right) X_{i}\left(H_{1} \otimes H_{2}\right) U^{\dagger}$, or $Z_{1}, Z_{2}$ and $X_{3}$ for $i=1,2$, and 3 , respectively. Again, each of $M_{i}$ is a two-qubit operator, but the following state

$$
|\psi\rangle=\frac{1}{\sqrt{2}}\left(\left|\psi_{+}\right\rangle+Q_{1}\left|\psi_{+}\right\rangle\right)=\frac{1}{\sqrt{2}}|0\rangle(|0\rangle+|1\rangle)|0\rangle
$$

can be prepared easily by measuring its stabilizers $Z_{1}, X_{2}$ and $Z_{3}$. Then we only need to measure a single twoqubit operator $M_{1}$ on $|\psi\rangle$ to obtain $\left|\psi_{+}\right\rangle$. Equivalently, we can also first prepare the state $|\psi\rangle=\frac{1}{\sqrt{2}}\left(I+Q_{2}\right)\left|\psi_{+}\right\rangle$ with stabilizers $X_{1}, Z_{2}$ and $Z_{3}$ or the state $|\psi\rangle=\frac{1}{\sqrt{2}}\left(I+Q_{3}\right)\left|\psi_{+}\right\rangle$with stabilizers $X_{1}, X_{2}$ and $X_{3}$, and measure the corresponding single stabilizer to obtain $\left|\psi_{+}\right\rangle$.

\section{Recursive preparation}

In this subsection, we will prove the following Theorem, which is used in Sec. III C:

Theorem 2: The encoded state $|\psi\rangle=U H^{\otimes n}\left(|0\rangle^{\otimes n}\right)$ for $U \in F_{k}$ can be prepared fault-tolerantly by recursive application of one-bit teleportation.

First we have the following Lemma, which is a generalization of Lemma 3.

Lemma 4: $|\psi\rangle$ can be created fault-tolerantly if given $\operatorname{St}(|\psi\rangle)=\left\{M_{1}, \ldots, M_{n}\right\}, \forall i, j$

(1) the cat-state-controlled- $M_{i}$ can be performed fault-tolerantly;

(2) there exists $Q_{i}$ such that $Q_{i}$ can be performed fault-tolerantly, $\left\{M_{i}, Q_{i}\right\}=0$, and for $i \neq j,\left[M_{i}, Q_{j}\right]=0$.

Proof: Since $M_{i}^{2}=I$, by applying the cat-state-controlled- $M_{i}$ and measuring the cat state fault-tolerantly as before, we can project any encoded state onto \pm 1 eigenstate of $M_{i}$. Then apply $Q_{i}$ if a -1 eigenstate is obtained. Using the same argument as in the proof of Lemma 3, we can fault-tolerantly prepare the state $|\psi\rangle$.

Lemma 5: If operations in $F_{k-1}$ and cat-state-controlled- $V$ for any $V \in F_{k-2}$ can be performed fault-tolerantly using the one-bit teleportation scheme, then $U H^{\otimes n}\left(|0\rangle^{\otimes n}\right)$ for $U \in F_{k}$ can be created fault-tolerantly.

Proof: The stabilizers of $|\psi\rangle=U H^{\otimes n}\left(|0\rangle^{\otimes n}\right)$ are $M_{i}=U H^{\otimes n} Z_{i}\left(U H^{\otimes n}\right)^{\dagger}=U X_{i} U^{\dagger}=U_{x} X_{i}$ for some $U_{x} \in F_{k-1}$. Define $Q_{i} \equiv U Z_{i} U^{\dagger}=Z_{i}$. $Q_{i}$ satisfies condition (2) of Lemma 4.

Since $M_{i}=U_{x} X_{i}$, the cat-state-controlled- $M_{i}$ is the product of cat-state-controlled- $U_{x}$ and cat-state-controlled$X_{i}$. The cat-state-controlled- $X_{i}$ is easily performed fault-tolerantly. Thus it remains to show how to perform the cat-state-controlled- $U_{x}$ for $U_{x} \in F_{k-1}$ fault-tolerantly.

As $U_{x} \in F_{k-1}$ is constructed with one-bit teleportation scheme using the circuit of Eq. (11), where $A_{i}=H, B_{i}=I$ and $D_{i}=X_{i}$, to perform cat-state-controlled- $U_{x}$, we need to perform cat-state-controlled- $E$, cat-state-controlled$U_{x} X_{i} U_{x}^{\dagger}$, and to prepare the ancilla $U_{x} H^{\otimes n}\left(|0\rangle^{\otimes n}\right)$ fault-tolerantly. As $E \in C_{2}$ and $U_{x} X_{i} U_{x}^{\dagger}=U_{x}^{\prime} X_{i}$ with $U_{x}^{\prime} \in F_{k-2}$, both of cat-state-controlled- $E$ and cat-state-controlled- $U_{x} D_{i} U_{x}^{\dagger}$ can be performed fault-tolerantly. Next, the state $U_{x} H^{\otimes n}|0\rangle^{\otimes n}$ has stabilizers $M_{i}^{\prime}=U_{x} X_{i} U_{x}^{\dagger}=U_{x}^{\prime} X_{i}$ with $U_{x}^{\prime} \in F_{k-2}$, which satisfies both conditions of Lemma 4 and can therefore be prepared fault-tolerantly. Thus cat-state-controlled- $U_{x}$ can be performed fault-tolerantly. This completes the proof of Lemma 5 .

In fact, what we have shown in the proof of Lemma 5 is that if operations in $F_{k-1}$ and the cat-state-controlled- $V$ for $V \in F_{k-2}$ can be performed fault-tolerantly, then the cat-state-controlled- $U$ for $U \in F_{k-1}$ and operations in $F_{k}$ 
can be performed fault-tolerantly. This is because according to Sec. III C, fault-tolerant construction of $F_{k}$ gates only require fault-tolerant $F_{k-1}$ gates and an ancilla $U H^{\otimes n}\left(|0\rangle^{\otimes n}\right)$ for $U \in F_{k}$.

Since both the operations in $F_{2}$ and the cat-state-controlled- $U$ for $U \in F_{1}$ can be performed fault-tolerantly, by induction, operations in $F_{k}$ and the cat-state-controlled- $U$ for $U \in F_{k-1}$ can be constructed fault-tolerantly, with which we can fault-tolerantly prepare the encoded state $U H^{\otimes n}\left(|0\rangle^{\otimes n}\right)$ for $U \in F_{k}$.

\section{APPENDIX C: ALTERNATIVE REMOTE CNOT CIRCUIT}

In this section, we re-derive the remote CNOT construction, given in [8], using one-bit teleportation. A remote CNOT between the states $|\alpha\rangle$ and $|\beta\rangle$ belonging to Alice and Bob, respectively, can be performed by a four step procedure: (1) Alice swaps her state $|\alpha\rangle$ with an ancilla $|0\rangle$, (2) Alice sends the teleported $|\alpha\rangle$ to Bob using $X$-teleportation, (3) Bob applies CNOT from $|\alpha\rangle$ to $|\beta\rangle$, and (4) Bob teleports $|\alpha\rangle$ back to Alice using $Z$-teleportation. Steps (2) and (4) involve prohibited operations. Here is a circuit representation:

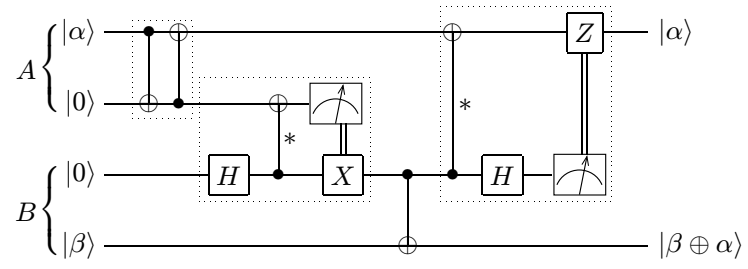

The two prohibited CNOTs are labelled with asterisks. They can be commuted backwards to obtain the equivalent circuit:

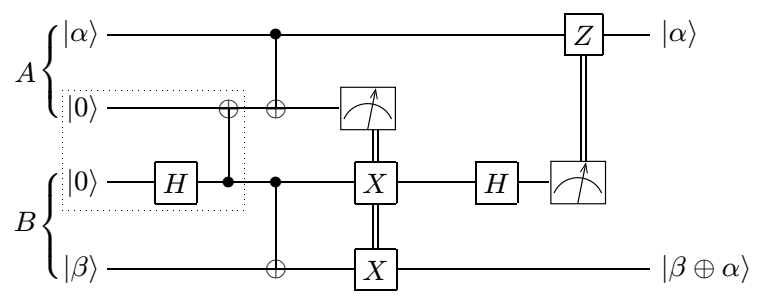

which again reduces prohibited operations to some specific shared entangled state.

[1] P. Shor, Proc. 37th Ann. Symp. on Found. of Computer Science, 56 (IEEE Comp. Soc. Press, 1996); LANL E-print quantu-ph/9605011.

[2] J. Preskill, Proc. Roy. Soc. A: Math., Phys. and Eng. 454, 385 (1998); LANL E-print quant-ph/9705031.

[3] E. Knill, R. Laflamme, and W. Zurek, Science 279, 342 (1998).

[4] A. M. Steane, Nature 399, 124 (1999).

[5] D. Gottesman, Phys. Rev. A 57, 127 (1998).

[6] P. O. Boykin, T. Mor, M. Pulver, V. Roychowdhury, and F. Vatan, Proc. 40th Ann. Symp. on Found. of Computer Science, 486 (IEEE Comp. Soc. Press, 1999); LANL E-print quant-ph/9906054.

[7] C. H. Bennett, G. Brassard, C. Crepau, R. Jozsa, A. Peres, and W. Wootters, Phys. Rev. Lett. 70,1895 (1993).

[8] D. Gottesman, Group 22: Proc. XXII International Colloquium on Group Theoretical Methods in Physics, eds. S. P. Corney, R. Delbourgo, and P. D. Jarvis, 32-43 (Cambridge, MA, International Press, 1999); LANL E-print quant-ph/9807006.

[9] D. Gottesman and I. L. Chuang, Nature 402, 390 (1999); LANL E-print quant-ph/9908010.

[10] We write $U E U^{\dagger}$ instead of $\left(U \otimes I^{\otimes n}\right) E\left(U^{\dagger} \otimes I^{\otimes n}\right)$ for simplicity. Unimportant identity operators are suppressed throughout the paper.

[11] P. Shor, SIAM J. Comp., 26, 1484 (1997).

[12] M. A. Nielsen and I. L. Chuang, Quantum computation and quantum information (Cambridge University Press, Cambridge, U.K., 2000).

[13] A. R. Calderbank and P. W. Shor, Phys. Rev. A 54, 1098 (1996).

[14] A. Steane, Proc. Roy. Soc. Lond. A 452, 2551 (1996). 
[15] A. Kitaev, unpublished.

[16] A. Kitaev, Russian Math. Surveys 52, 1191 (1997).

[17] D. DiVincenzo and P. Shor, Phys. Rev. Lett. 77, 3260 (1996)

[18] Note that the $n$ operators $U D_{i} U^{\dagger}$ to be performed conditioned on the measurement outcomes are precisely the $n$ stabilizers of the ancilla state $U A\left(|0\rangle^{\otimes n}\right)$. This coincidence is due to the structure of the teleportation circuits. See also the examples in Sec. IV.

[19] D. Gottesman (private communication).

[20] D. Coppersmith, IBM Research Report RC 19642 (1994).

[21] G. Brassard, S. Braunstein, and R. Cleve, Physica D. 120, 43 (1998).

[22] R. Cleve and H. Buhrman, Phys. Rev. A 56, 1201 (1997).

[23] R. Cleve, W. van Dam, M. Nielsen, and A. Tapp, Proc. of the 1st NASA International Conference on Quantum Computing and Quantum Communications, 61 (1999); LANL E-print quant-ph/9708019.

[24] J. L. Hennessey, D. Goldberg, and D. A. Patterson, Computer Architecture : A Quantitative Approach (Academic Press, New York, 1996). 